\title{
Allergenicity and toxicology of inhaled silver nanoparticles in allergen-provocation mice models
}

This article was published in the following Dove Press journal:

International Journal of Nanomedicine

21 November 2013

Number of times this article has been viewed

\author{
Hsiao-Chi Chuang ${ }^{1,2, *}$ \\ Ta-Chih Hsiao,* \\ Cheng-Kuan $\mathrm{Wu}^{4}$ \\ Hui-Hsien Chang ${ }^{5}$ \\ Chii-Hong Lee 6 \\ Chih-Cheng Chang ${ }^{1,2}$ \\ Tsun-Jen Cheng ${ }^{4,7}$
}

\section{On behalf of the Taiwan \\ CardioPulmonary Research \\ Group (T-CPR)}

'School of Respiratory Therapy,

College of Medicine, ${ }^{2}$ Division of

Pulmonary Medicine, Department

of Internal Medicine, Shuang Ho

Hospital, Taipei Medical University,

Taipei, ${ }^{3}$ Graduate Institute of

Environmental Engineering, National

Central University, Taoyuan, ${ }^{4}$ Institute of Occupational Medicine and Industrial Hygiene, College of Public Health, National Taiwan University, Taipei, ${ }^{5}$ Institute of Environmental Health, College of Public Health, National Taiwan University,

${ }^{6}$ Department of Pathology, Shuang Ho Hospital, Taipei Medical University, Taipei, ${ }^{7}$ Department of Public Health, College of Public Health, National Taiwan University, Taipei, Taiwan

*These authors contributed equally to this work

Correspondence: Tsun-Jen Cheng Institute of Occupational Medicine and Industrial Hygiene and Department of Public Health, College of Public Health, National Taiwan University, No. 17, Shiujou Rd, Taipei, Taiwan

$\mathrm{Tel}+886233668090$

Fax + 886223957845

Email tcheng@ntu.edu.tw

\begin{abstract}
Silver nanoparticles (AgNP) have been associated with the exacerbation of airway hyperresponsiveness. However, the allergenicity and toxicology of AgNP in healthy and allergic individuals are unclear. We investigated the pathophysiological responses to AgNP inhalation in a murine model of asthma. Continuous and stable levels of $33 \mathrm{~nm} \mathrm{AgNP}$ were maintained at $3.3 \mathrm{mg} / \mathrm{m}^{3}$ during the experimental period. AgNP exposure concomitant with ovalbumin challenge increased the enhanced pause (Penh) in the control and allergic groups. AgNP evoked neutrophil, lymphocyte and eosinophil infiltration into the airways and elevated the levels of allergic markers (immunoglobulin $\mathrm{E}[\mathrm{IgE}]$ and leukotriene $\mathrm{E}_{4}\left[\mathrm{LTE}_{4}\right]$ ), the type $2 \mathrm{~T}$ helper (Th2) cytokine interleukin-13 (IL-13), and oxidative stress (8-hydroxy-2'-deoxyguanosine [8-OHdG]) in healthy and allergic mice. Bronchocentric interstitial inflammation was observed after AgNP inhalation. After inhalation, the AgNP accumulated predominantly in the lungs, and trivial amounts of AgNP were excreted in the urine and feces. Furthermore, the AgNP induced inflammatory responses in the peritoneum. The inhalation of AgNP may present safety concerns in healthy and susceptible individuals.
\end{abstract}

Keywords: inductively coupled plasma-mass spectrometry, inflammation, leukotriene E4, ovalbumin, oxidative stress

\section{Introduction}

Asthma is a hyperresponsive airway disease in humans and is a critical public health concern that has become increasingly prevalent. It is estimated that 300 million people worldwide suffer from asthma, ${ }^{1}$ and this number is expected to increase to 400 million by $2025 .{ }^{2}$ The risk factors for asthma development can be categorized into host and environmental factors. ${ }^{3}$ Epidemiological studies have demonstrated an association between the incidence of allergic diseases and exposure to ambient particulate matter (PM) ${ }^{4,5} \mathrm{PM}$ can exert adverse effects on the respiratory tract, which may increase the prevalence of allergic diseases associated with the physicochemistry of PM ${ }^{6,7}$ Recent studies have indicated that nano-scaled PM, particularly nanoparticles (NP), induce immunotoxic and allergic responses, ${ }^{8,9}$ which represent a new and plausible health risk for healthy and asthmatic individuals.

An increasing number of studies have focused on the biomedical applications of NPs because of their distinct physicochemical characteristics. ${ }^{10}$ Metal-based NPs, for example, have an important application as nano-sized sensors for biological labeling and as vectors for drug delivery. ${ }^{11,12}$ However, many metal NPs, such as zinc oxide NPs, have adverse effects on human health because of their high bioreactivity. ${ }^{13,14}$ A number of distinct metal NPs, such as silver NPs (AgNP), are known to reduce 
inflammation and exhibit antibacterial activities, ${ }^{15}$ suggesting that AgNP have inflammatory effects and aid wound healing. ${ }^{15,16}$ Recently, studies have discussed the possibility of using AgNP to treat asthma; ${ }^{16,17}$ however, the allergenicity and toxicity of AgNP inhalation in healthy and asthmatic individuals are unclear.

To investigate the effects of AgNP in healthy and allergic individuals, we established an ovalbumin (OVA)-sensitized allergic mouse model. We used an evaporation-condensation method to generate AgNP, which were inhaled by allergic and healthy mice. After AgNP inhalation, enhanced pause (Penh), and allergic responses, concurrent with the induction of oxidative stress and inflammation in the pulmonary-tosystemic system, were examined in the allergic and healthy mice. Penh is an index of airway hyperreactivity that has been used in previous allergy studies. ${ }^{16,18}$ In addition, we evaluated the excretion and distribution of AgNP and examined their systemic tissue distribution after inhalation. The allergenicity and toxicity of inhaled AgNP were determined by evaluating allergic responses and inflammatory and oxidative potencies, which play a role in regulating the immune system.

\section{Materials and methods}

\section{AgNP generation system}

In this study, we used an evaporation-condensation method to generate the AgNP used for inhalation in mice. The design of this system has been described previously. ${ }^{19,20}$ Briefly, approximately $3 \mathrm{~g}$ of silver powder (>99\%, Merck KGaA, Darmstadt, Germany) was evaporated at the center of a $1,100^{\circ} \mathrm{C}$ tube furnace (Model T11-301, SJ Ltd, Taipei, Taiwan; Table 1), and the generated silver vapor was transported continuously using nitrogen at a flow rate of $6 \mathrm{~L} /$ minute. Because the outlets of the furnace were equipped with a chilling system, the silver vapor

Table I Operation parameters for generating AgNP using the evaporation-coagulation AgNP generation system and AgNP characterization in the whole-body exposure chamber

\begin{tabular}{ll}
\hline & Mean (SD) \\
\hline Operation conditions & \\
Furnace temperature $\left({ }^{\circ} \mathrm{C}\right)$ & 1,100 \\
Nitrogen air $(\mathrm{L} / \mathrm{min})$ & 6 \\
Nitrogen and oxygen air $(\mathrm{I}: \mathrm{I} ; \mathrm{L} /$ minute) & 5 \\
AgNP characterization & \\
GMD $(\mathrm{nm})$ & 33 \\
GSD & 2.1 \\
Number concentration $\left(\mathrm{L} / \mathrm{cm}^{3}\right)$ & $1.4 \times 10^{7}\left(7.0 \times 10^{4}\right)$ \\
Mass concentration $\left(\mathrm{mg} / \mathrm{m}^{3}\right)$ & $3.3(0.7)$ \\
Surface area concentration $\left(\mathrm{mm}^{2} / \mathrm{m}^{3}\right)$ & $1.6 \times 10^{4}\left(2.7 \times 10^{3}\right)$ \\
\hline
\end{tabular}

Notes: Values are expressed as the mean (SD); $n=7$.

Abbreviations: AgNP, silver nanoparticles; GMD, geometric mean diameter; GSD, geometric standard deviation; SD, standard deviation. was quenched suddenly and condensed to form the AgNP. In this study, an additional condition section was introduced between the generation system and the exposure chamber to guarantee that the temperature, relative humidity, and composition of the exposed AgNP stream were close to the constant environmental conditions. The oxygen and nitrogen air $(5 \mathrm{~L} /$ minute at a flow rate of 1:1; Table 1) was delivered and mixed with the exposed AgNP stream. The oxygen level was maintained at approximately $21 \%$ throughout the exposure period. Next, the exposed AgNP stream was split into two routes for filtration with or without high-efficiency particulate air (HEPA) filters before its introduction into the whole-body exposure chambers. The exposure chamber has been described previously. ${ }^{21}$

\section{AgNP characterization}

A TSI scanning mobility particle sizer with nano-DMA (model 3936, TSI Incorporated, Shoreview, MN, USA) was used to monitor the size distribution of the AgNP in the exposure chamber continuously throughout the entire exposure period. The flow rates of the aerosol stream and sheath air of the scanning mobility particle sizer were fixed at $0.3 \mathrm{~L} /$ minute and $3 \mathrm{~L} /$ minute, respectively. The scanning mobility particle sizer was calibrated before the experiment using 100-nm National Institute of Standards and Technology (NIST)-traceable PSL (Polystyrene Latex Spheres) standard particles. The total particle number concentration, the number-based geometric mean diameter (GMD), and the geometric standard deviation (GSD) were recorded. The total surface area was estimated using TSI Inc software (Aerosol Instrument Manager, AIM 9.0) under the assumption of spherical particles. We collected real-time scanning mobility particle sizer measurements of the generated AgNP every 5 minutes during exposure. To physicochemically characterize the generated AgNP, they were collected onto $37 \mathrm{~mm}$ Teflon substrates (Merck Millipore, Darmstadt, Germany), quarters of which were fixed onto $13 \mathrm{~mm}$ aluminum scanning electron microscopy (SEM) stubs. ${ }^{22}$ The samples were platinum $(\mathrm{Pt})$ coated to an average thickness of $10 \mathrm{~nm}$ using a sputter coater and were imaged using an Inspect ${ }^{\mathrm{TM}}$ SEM (FEI Company, Hillsboro, OH, USA) at an accelerating voltage of $3 \mathrm{kV}$ and a spot size of 2.5. X-ray microanalysis was performed using an energy-dispersive X-ray spectroscopy (EDX) analysis Genesis System.

\section{Animals}

Female BALB/c mice (6 weeks old) were obtained from BiOLASCO Taiwan Co., Ltd (Taipei, Taiwan). The mice were maintained at a constant temperature and relative humidity of $22^{\circ} \mathrm{C} \pm 2{ }^{\circ} \mathrm{C}$ and $55 \% \pm 10 \%$, respectively, with 
a light/dark cycle (12 hour/12 hour) throughout the study. The mice weighed between $17 \mathrm{~g}$ and $22 \mathrm{~g}$ during the experimental period. The animals were housed in plastic cages and were provided Lab Diet 5001 (PMI Nutrition International, St Louis, MI, USA) and water ad libitum during acclimatization as well as pre-exposure and post-exposure. The animal experiments were performed in compliance with the animal and ethics review committee of the Laboratory Animal Centre at the National Taiwan University (Taiwan).

\section{Allergic sensitization, AgNP inhalation, and OVA challenge}

To assess the effects of AgNP on allergic responses, we used endotoxin-free OVA (Sigma-Aldrich, St Louis, MI, USA) as an allergen to induce systemic sensitization. The development of allergic airway disease in mice after AgNP exposure was modified from a previously published method. ${ }^{23}$ The experimental design is shown in Figure 1. On day 0, the mice in the allergic group received an intraperitoneal injection of $50 \mu \mathrm{g}$ of OVA in aluminum hydroxide adjuvant, whereas those in the control group received the same volume of adjuvant alone. On day 8 , the mice in the allergic group received an intraperitoneal injection of $25 \mu \mathrm{g}$ of OVA in aluminum hydroxide adjuvant, whereas the control group received the same volume of adjuvant alone. The mice in the control group demonstrated a significantly lower level of OVA-specific IgE than the mice sensitized with OVA (Figure 2A, $P<0.001$ ). On day 15, the baseline of Penh was determined before AgNP exposure and OVA challenge. On day 16, the allergic and control mice were divided into two subgroups as follows: 1 ) mice exposed to AgNPs (Ag; $\mathrm{n}=6$ ), and 2) mice exposed to
HEPA-filtered air (FA; n=5); exposure occurred for 7 days (6 hours/day, from day 16 to 22) for both subgroups. After exposure to $\mathrm{Ag} / \mathrm{FA}$, the mice were challenged intranasally with $100 \mu \mathrm{g}$ of OVA between days 23 and 25, and the Penh was examined on day 26 . The animals were euthanized (six mice in the AgNP exposure subgroup and five mice in the FA control subgroup) on day 27 , and bronchoalveolar lavage fluid (BALF), blood, and tissue samples were collected. In addition, urine and feces were collected between days 16 and 22 (18 hours/day for 7 days) using metabolic cages (Harvard Apparatus, Holliston, MA, USA).

\section{Determination of Penh}

A whole-body plethysmograph system (Buxco Electronics, Inc., Wilmington, NC, USA) was used to determine the immediate Penh before and after AgNP exposure and the Penh to methacholine aerosol exposure in unanesthetized and unrestrained mice. The calibration and principles of Penh measurement using this system have been described in previous studies. ${ }^{18,24,25}$ After acclimatization of the mice in the chamber for 5 minutes, the airway responses were measured for 3 minutes, which represented the baseline response before exposure to phosphate-buffered saline (PBS) or methacholine. PBS or methacholine $(5,10$, and $25 \mathrm{mg} / \mathrm{mL})$ was nebulized through an inlet in the main chamber for 3 minutes. The Penh was measured for 3 minutes after exposure to PBS or methacholine, and the signals representing airway reactivity were recorded in accordance with previous studies. ${ }^{18,24}$ Penh reflects changes in the waveform of the chamber pressure signal from both inspiration and expiration.

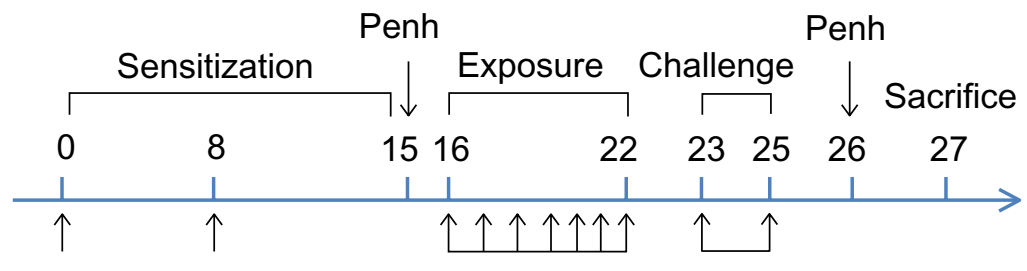

$\begin{array}{lccll}\text { Control (C) } & & & \\ \text { Group 1 (FA-C) } & \text { PBS } & \text { PBS } & \text { HEPA-filtered air } & 100 \mu \mathrm{g} \text { OVA } \\ \text { Group 2 (Ag-C) } & \text { PBS } & \text { PBS } & 3.3 \mathrm{mg} / \mathrm{m}^{3} \mathrm{AgNP} & 100 \mu \mathrm{g} \text { OVA } \\ \text { Allergy (A) } & & & \\ \text { Group 3 (FA-A) } & 50 \mu \mathrm{g} \text { OVA } 25 \mu \mathrm{g} \text { OVA } & \text { HEPA-filtered air } & 100 \mu \mathrm{g} \text { OVA } \\ \text { Group 4 (Ag-A) } & 50 \mu \mathrm{g} \text { OVA } 25 \mu \mathrm{g} \text { OVA } & 3.3 \mathrm{mg} / \mathrm{m}^{3} \mathrm{AgNP} & 100 \mu \mathrm{g} \text { OVA }\end{array}$

Figure I Experimental design for investigating the effects of AgNP on the allergic response to OVA antigen.

Notes: OVA in aluminum hydroxide adjuvant is prepared in PBS; PBS (control) is aluminum hydroxide adjuvant prepared in PBS.

Abbreviations: A, allergy; AgNP, silver nanoparticles; C, control; FA, filtered air; HEPA, high-efficiency particulate air; OVA, ovalbumin; PBS, phosphate-buffered saline; Penh, enhanced pause. 
A
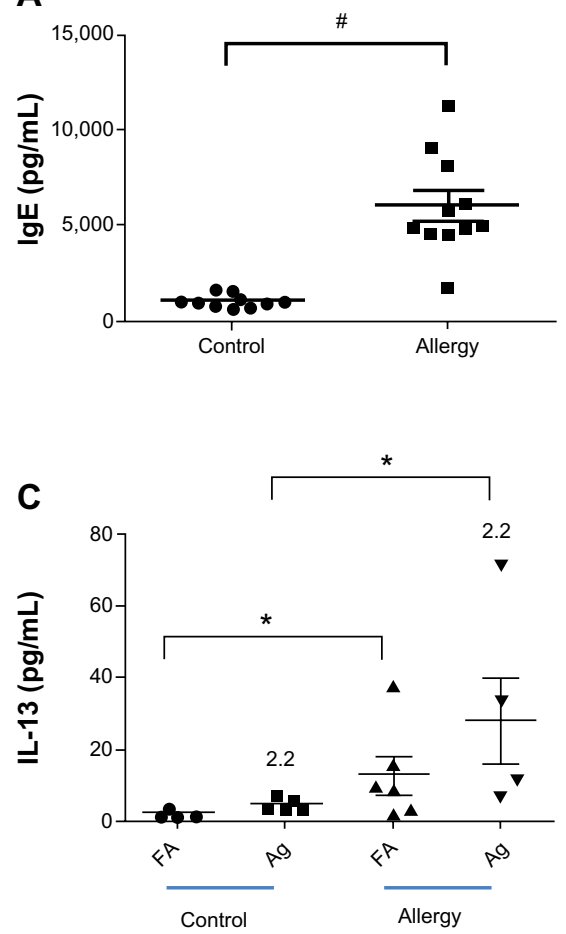

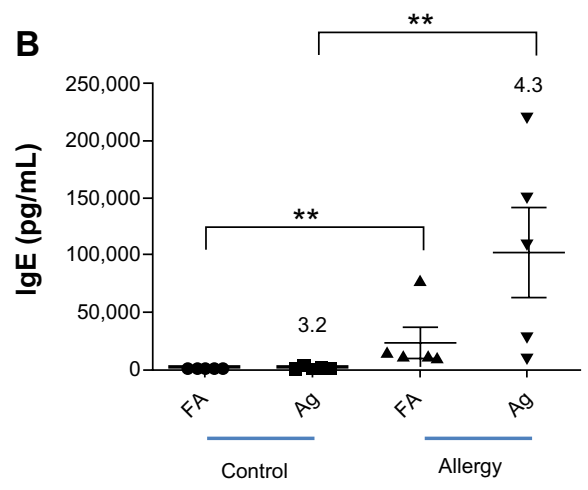

D

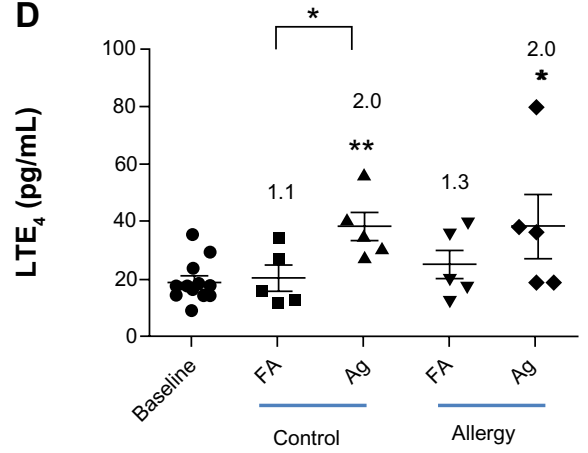

Figure 2 OVA-specific IgE was collected (A) I day before and (B) 2 days after AgNP/FA inhalation and OVA challenge in the control and allergic mice. The effects of AgNP/ FA on (C) IL-I 3 levels in the BALF and (D) $\mathrm{LTE}_{4}$ levels in the urine were determined in control and allergic mice.

Notes: Values are expressed as the mean \pm standard deviation; $n=5-6$. ${ }^{*} P<0.00$ I; $* * P<0.01 ; * P<0.05$.

Abbreviations: AgNP, silver nanoparticles; BALF, bronchoalveolar lavage fluid; FA, filtered air; lgE, immunoglobulin E; IL, interleukin; LTE ${ }_{4}$, leukotriene $E_{4}$; OVA, ovalbumin.

\section{Animal necropsy, sample collection, and analysis}

Animal necropsy and BALF, blood, and tissue collections were performed as described previously. ${ }^{6}$ Briefly, animals were euthanized with a single intraperitoneal injection of $2 \mathrm{~mL}$ of sodium pentobarbitone $(200 \mathrm{mg} / \mathrm{mL})$ after the determination of Penh. A single $1 \mathrm{~mL}$ volume of PBS was used to lavage the lungs. We retained this first BALF in a separate tube to analyze biochemical markers. Subsequently, we lavaged the lungs a further three times with $1 \mathrm{~mL}$ volumes of PBS. All samples were centrifuged at 1,500 $\times \mathrm{g}$ for 5 minutes at $4{ }^{\circ} \mathrm{C}$, the supernatant was removed, and the cell pellet from the first lavage was combined with the cells from the subsequent lavage before resuspension in $1 \mathrm{~mL}$ of PBS. Cytospin preparations were made using 10,000 cells centrifuged at $15 \times \mathrm{g}$ for 3 minutes onto glass slides. Two hundred cells were counted per slide. The differential cells in the BALF samples were treated with Liu's stain (ASK, Taipei, Taiwan) and identified under light microscopy. ${ }^{26}$ The differential cell counts were presented in percentages of the total number of cells. The plasma OVA-specific $\operatorname{IgE}$ levels before (on day 15) and after (on day 23) AgNP exposure and OVA challenge were measured using an enzyme- linked immunosorbent assay (ELISA; R\&D Systems, Inc, Minneapolis, MN, USA). ${ }^{18}$ The proinflammatory cytokine interleukin-13 (IL-13) was quantified in the BALF samples using ELISA in accordance with the manufacturer's instructions (R\&D Systems).

\section{Urinary leukotriene $\mathrm{E}_{4}$ and 8-hydroxy-2'-deoxyguanosine}

Urine and feces samples were collected from each mouse during the 8 consecutive exposure days (between days 15 and 22). Samples were collected every 18 hours post-exposure. The urinary leukotriene $\mathrm{E}_{4}\left(\mathrm{LTE}_{4}\right)$ level was measured using an enzyme immunoassay (Cayman Chemical Company, Ann Arbor, MI, USA) in accordance with the manufacturer's instructions. The principle of the immunoassay has been described previously in a clinical study. ${ }^{27}$ Briefly, the assay is based on the competition between $\mathrm{LTE}_{4}$ and an $\mathrm{LTE}_{4}$-acetylcholinesterase conjugate for a limited amount of $\mathrm{LTE}_{4}$ antiserum. A PowerWave microplate reader (BioTek Instruments, Inc., Winooski, VT, USA) was used to determine the absorbance of $\mathrm{LTE}_{4}$ at $410 \mathrm{~nm}$. In addition, an ELISA kit (JaICA, Tokyo, Japan) was used to determine the oxidative DNA damage, which was assessed by measuring the urinary 
8-hydroxy-2'-deoxyguanosine (8-OHdG) levels; the absorbance was measured on a microplate reader at $450 \mathrm{~nm}$. The excretion ratio was used to adjust the urinary $8-\mathrm{OHdG}$ levels by urination volume, collection hour, and body weight and was expressed as the $8-\mathrm{OHdG}$ excretion ratio $\left(\mathrm{ng} \cdot \mathrm{hour}^{-1} \cdot \mathrm{kg}^{-1}\right)$, in accordance with the manufacturer's instructions.

\section{AgNP in urine, feces and tissues}

The AgNP concentrations in the urine, feces, and tissues (ie, the brain, lung, heart, liver, spleen, and kidney) were determined using inductively coupled plasma-mass spectrometry (ICP-MS; Agilent 7500C, Agilent Technologies, Santa Clara, CA, USA). The feces and tissue samples were lyophilized to obtain the dry weight. The lyophilized samples were digested using concentrated nitric acid (Thermo Fisher Scientific, Waltham, MA, USA) in a MARS 5 microwave system (CEM Corporation, Matthews, NC, USA) in CEM advanced Teflon-lined composite vessels. ${ }^{28}$ The digestion temperature was increased to $150^{\circ} \mathrm{C}$ for 30 minutes. Nitric acid and deionized (>18 M $\Omega$ ) water were added to the urine, feces, and tissue samples to a final concentration of 5\% nitric acid. Nitric acid and deionized water blanks were used to detect contamination during the analysis process. A NIST standard reference material (SRM) solution was used to check the accuracy of the analyses. The recovery rate and coefficient of variation for the SRM were $97.6 \%$ and $15.7 \%$, respectively.

\section{Histological analysis}

For histological analysis, the brain, lungs, heart, liver, spleen, and kidneys were excised and washed with ice-cold PBS. Each organ was fixed in 10\% neutral buffered formalin and embedded in paraffin, sectioned, and stained with hematoxylin and eosin (H\&E). A histopathologist examined the histological samples in a blinded fashion under a light microscope.

\section{Data analysis}

The statistical analyses were performed using GraphPad version 5 (GraphPad Software, Inc., La Jolla, CA, USA) for Windows. The Mann-Whitney $U$-test was used for comparisons between groups. ${ }^{29}$ The level of significance was set at $P<0.05$.

\section{Results \\ Characterization of AgNP}

The AgNP spheres generated using the evaporation-condensation method were physicochemically characterized using an SEM equipped with EDX. Comparison of the EDX spectrum of the filter with and without collecting the AgNP demonstrated that only silver-containing NPs were produced by the system (Figure 3A) along with the background elemental signals from the Teflon substrate (carbon $[\mathrm{C}]$ and fluorine $[\mathrm{F}]$ ) and coating material $(\mathrm{Pt})$. The SEM-EDX results excluded the possibility that other uncertain effects were introduced by
A
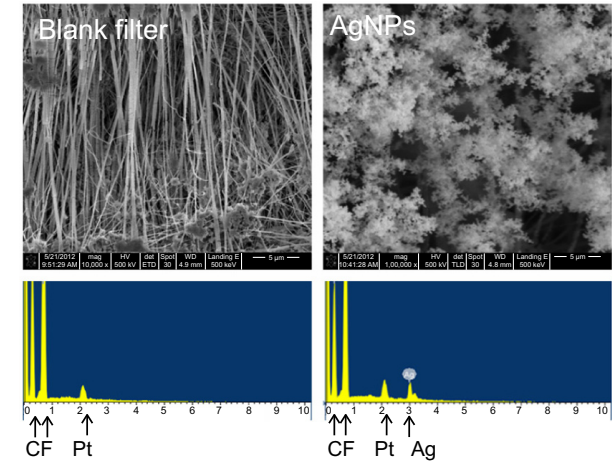

C

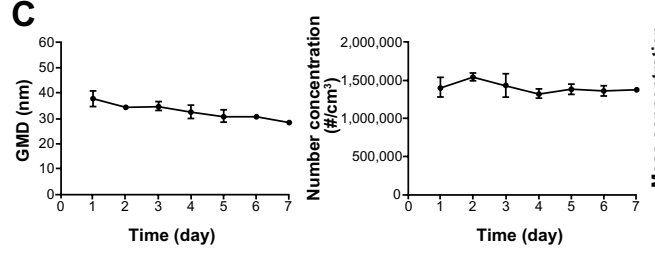

B
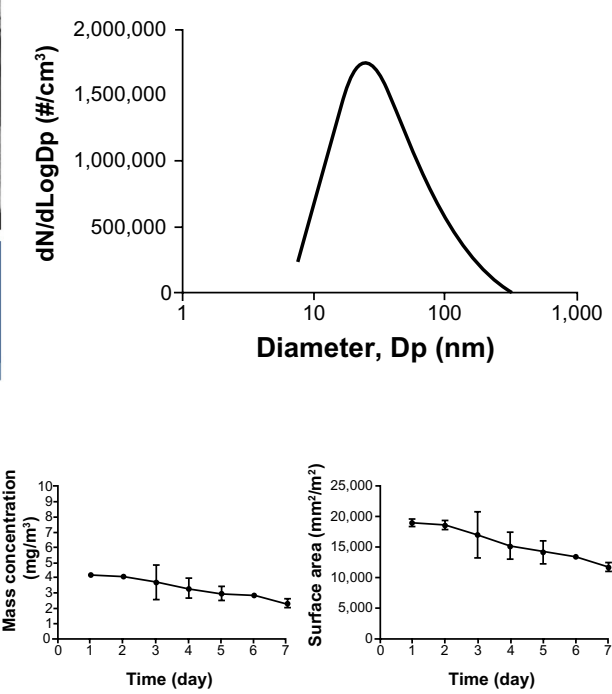

Figure 3 (A) Scanning electron microscopy images of AgNP collected from the evaporation-coagulation AgNP generation system and EDX data of the blank Teflon substrate and AgNP collected on the Teflon substrate. (B) Number-size distribution of AgNP produced by the evaporation-coagulation system. (C) Daily change in average GMD, number concentration, and mass concentration of AgNP measured in the whole-body exposure chamber.

Abbreviations: AgNP, silver nanoparticles; EDX, energy-dispersive X-ray spectroscopy; GMD, geometric mean diameter. 
impurities or contamination during the AgNP generation processes. The AgNP produced using this system demonstrated a normal number-size distribution (Figure 3B). In Figure 3C, the geometric mean diameter and the daily average exposure concentrations of the AgNP based on their number, mass, and surface area, as determined by SMPS, are presented. The corresponding numerical average values (standard deviation [SD]) were $33 \mathrm{~nm}$ GMD (2.1 for GSD), $1.4 \times 10^{7}\left(7.0 \times 10^{4}\right)$ $\# / \mathrm{cm}^{3}$ for number concentration, $3.3(0.7) \mathrm{mg} / \mathrm{m}^{3}$ for mass concentration, and $1.6 \times 10^{4}\left(2.7 \times 10^{3}\right) \mathrm{mm}^{2} / \mathrm{m}^{3}$ for surface area concentration (Table 1). The total number concentration was stable, although the other three parameters of the AgNP distributions decreased slightly over the exposure period (7 days; Figure 3C). In general, the AgNP generated and delivered to the whole-body exposure chamber were stable and uniform in size, number, and mass concentration and surface area over the exposure period.

\section{AgNP increased Penh}

A significant Penh response to methacholine was not observed in the allergic and control groups before AgNP exposure (Figure 4A). After AgNP exposure and OVA challenge, dose-dependent responses to methacholine were observed in the allergic and control groups (Figure 4B). In the allergic mice treated with $25 \mathrm{mg} / \mathrm{mL}$ methacholine, AgNP exposure increased the Penh levels 14.5-fold compared to the baseline values $(P<0.001$; Figure $4 \mathrm{C})$; exposure of the allergic mice to FA increased the Penh levels 9.5-fold compared with the baseline values $(P<0.001)$. Exposure of the control mice to the AgNP and FA increased the Penh levels 8.2-fold $(P<0.001)$ and 4.7-fold $(P<0.001)$, respectively, compared to the baseline levels for $25 \mathrm{mg} / \mathrm{mL}$ methacholine. Following the administration of PBS, significant changes in the Penh compared with baseline were not observed. Furthermore, there was no significant increase in the Penh levels after AgNP exposure compared to the Penh levels after FA exposure in the allergic and control mice.

The Penh responses in the allergic mice were 11.2-fold higher than before AgNP exposure and OVA challenge $(P<0.01$; Figure 4D) and 9.2-fold higher than before FA exposure and OVA challenge $(P<0.01)$. The control mice exhibited a similar increase in Penh levels; however, this increase was lower than observed in the allergic mice, ie, 7.5-fold higher than before AgNP exposure $(P<0.01)$ and 6.7-fold higher than before FA exposure $(P<0.01$; Figure 4D).

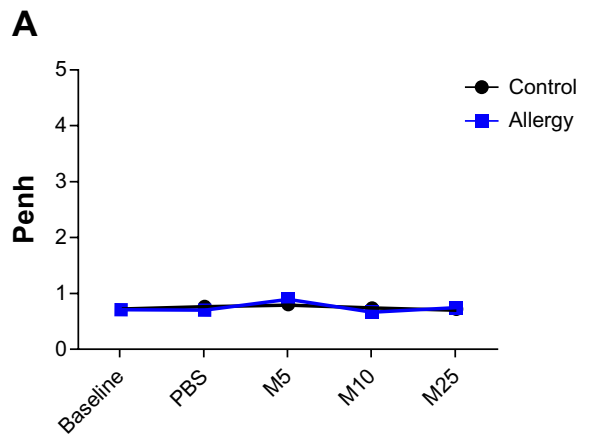

B
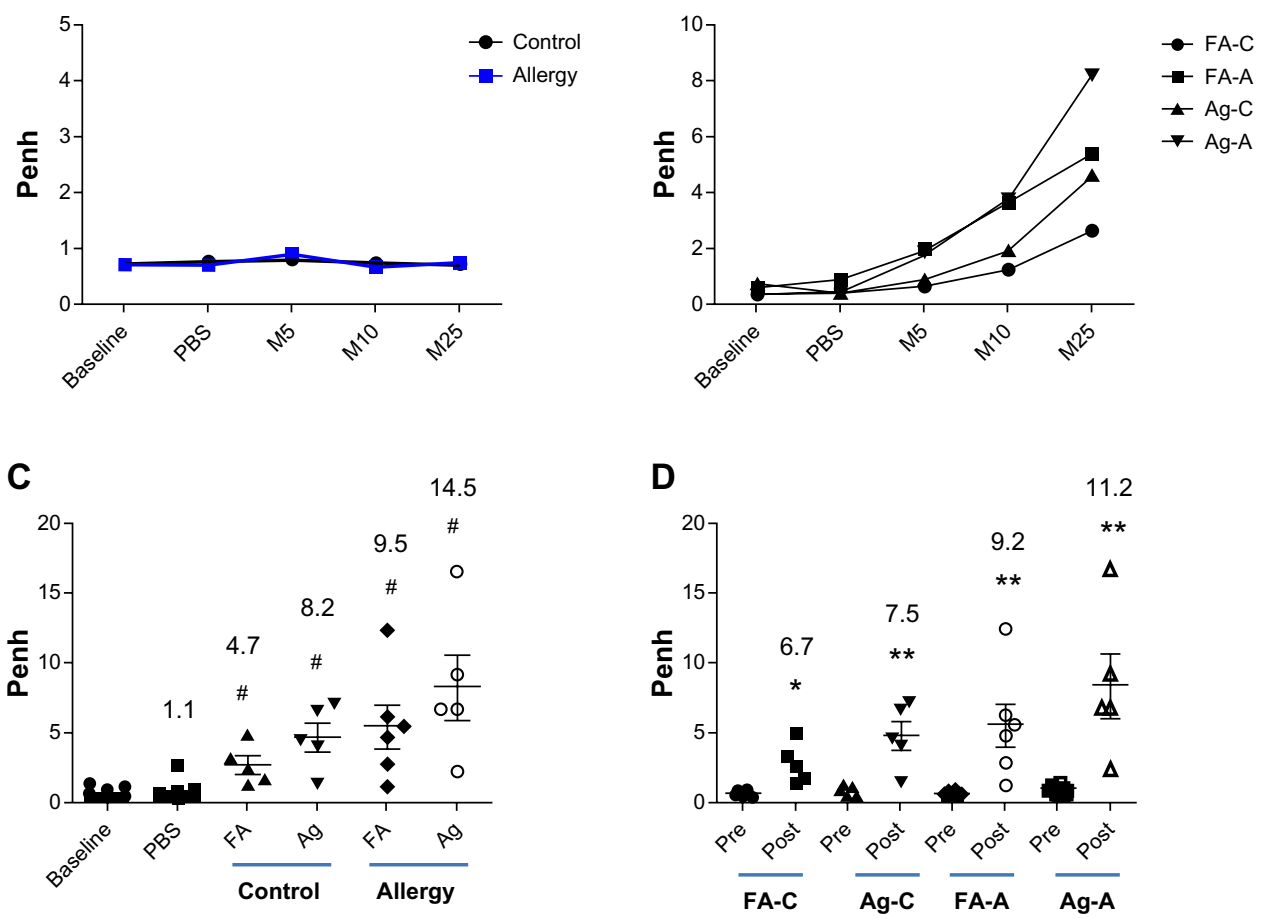

Figure 4 Penh response to aerosolized methacholine measured (A) I day before AgNP/FA exposure and OVA challenge, and (B) I day after AgNP/FA exposure and OVA challenge in the control (FA-C and Ag-C) and allergic mice (FA-A and Ag-A). Penh response to 5 (M5), 10 (MI0), and $25 \mathrm{mg} / \mathrm{mL}$ methacholine (M25) measured (C) I day after AgNP/FA exposure and OVA challenge, and (D) I day before and after AgNP/FA exposure and OVA challenge in the control and allergic mice.

Notes: Values are expressed as the mean \pm standard deviation; $n=5-6$. ${ }^{*} P<0.001 ; * * P<0.01 ; * P<0.05$

Abbreviations: A, antigen; AgNP, silver nanoparticles; C, control; FA, filtered air; M, methacoline; OVA, ovalbumin; PBS, phosphate-buffered saline; Penh, enhanced pause. 


\section{Effects of inhaled AgNP on allergic development}

A slight increase in the percentage of neutrophils and lymphocytes were observed in the BALF of the allergic and control mice after $3.3 \mathrm{mg} / \mathrm{m}^{3}$ AgNP exposure and OVA challenge compared to after FA exposure (Figure 5A and B); however, these differences were not statistically significant. In the control mice, a significant increase in the percentage of eosinophils was observed after AgNP exposure compared to after FA exposure ( $P<0.001$; Figure 5C), whereas a significant difference in the eosinophil percentage was not observed in the allergic mice after the AgNP and FA exposures. By contrast, the eosinophil percentage was significantly higher in the allergic mice than in the control mice after the AgNP and FA exposures $(P<0.05$; Figure 5C). When exposed to AgNP, both groups of mice, particularly the control mice, demonstrated a decrease in the percentage of BALF macrophages compared to the mice exposed to FA $(P<0.05$, Figure 5D). Moreover, the allergic mice exposed to FA exhibited significantly lower macrophage levels than the control mice $(P<0.05$; Figure 5D). The histological observations were consistent with the cytological results, which showed that lymphocytic and neutrophilic inflammatory infiltration was present in the control mice exposed to AgNP and the allergic mice exposed to FA or AgNP (Figure 5E); this infiltration was more prominent in the control mice exposed to the AgNP. In particular, interstitial inflammation occurred in the bronchial center (Figure 5E), with eosinophils and macrophages infiltrating the lungs, particularly in the allergic mice.

After exposure to the AgNP, the allergic mice demonstrated higher levels of allergic and inflammatory responses than the control mice, as determined by the serum levels of OVA-specific $\operatorname{IgE}(P<0.05$, Figure $2 \mathrm{~B})$ and the BALF levels of IL-13 $(P<0.05$, Figure $2 \mathrm{C})$, respectively, in samples collected 2 days after OVA challenge. In the allergic mice, AgNP inhalation resulted in IgE levels that were 4.3-fold higher and IL-13 levels that were 2.2-fold higher than after FA inhalation (Figure $2 \mathrm{~B}$ and $\mathrm{C}$ ). In the control mice, AgNP exposure produced IgE and IL-13 responses similar to those in the allergic mice, which were 3.2-fold higher and 2.2-fold higher, respectively, compared to after FA exposure.

A significant difference in the $\mathrm{LTE}_{4}$ level was not observed in the ratios of allergic mice to control mice after the AgNP and FA exposures over the 7-day period. On the last day of exposure, the AgNP induced a 2-fold increase in the $\mathrm{LTE}_{4}$ levels compared to the baseline levels before exposure in both the allergic and control mice (Figure 2D). In addition, after FA exposure, the $\mathrm{LTE}_{4}$ levels were 1.1-fold higher than baseline in the control mice and 1.3-fold higher than baseline in the allergic mice (Figure 2D). Differences were only observed in the control mice with respect to the AgNP and FA exposures $(P<0.05$; Figure 2D).

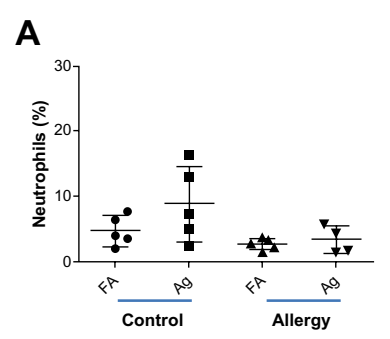

E

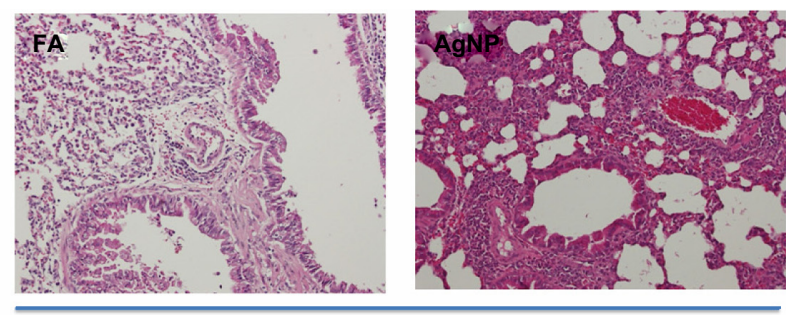

Control

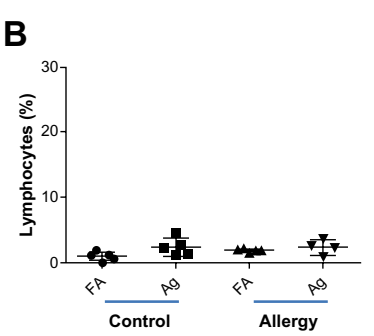

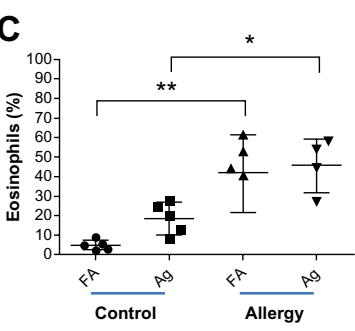
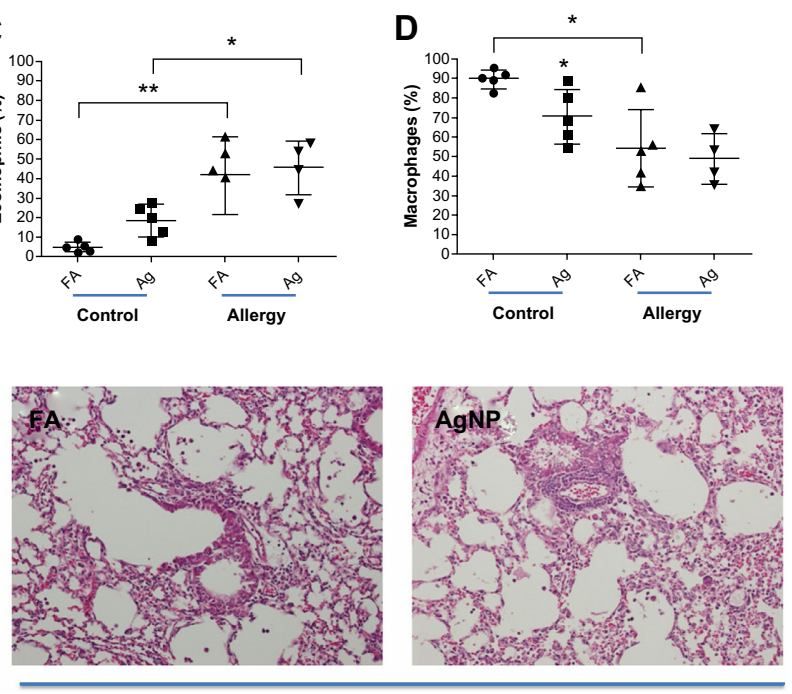

Allergy

Figure 5 Percentages of (A) BALF neutrophils, (B) lymphocytes, (C) eosinophils, and (D) macrophages recovered 2 days after AgNP/FA exposure and OVA challenge in control and allergic mice. (E) Photomicrographs $(200 \times)$ of hematoxylin and eosin staining of the lungs from the control and allergic mice after AgNP/FA exposure and OVA challenge. In the control mice, AgNP exposure induced lymphocytic and neutrophilic inflammatory infiltration; in the allergic mice, AgNP/FA exposure resulted in lymphocytic and neutrophilic inflammation as well as the recruitment of eosinophils and macrophages into the lungs.

Notes: Values are expressed as the mean \pm standard deviation; $n=5-6$. ${ }^{*} \mathrm{P}<0.0 \mathrm{I} ; * \mathrm{p}<0.05$.

Abbreviations: AgNP, silver nanoparticles; BALF, bronchoalveolar lavage fluid; FA, filtered air; OVA, ovalbumin. 


\section{Oxidative stress induced by AgNP}

Throughout the exposure period, AgNP exposure resulted in a slight increase in the $8-\mathrm{OHdG}$ levels in the allergic mice compared to the control mice, after adjustment for the levels during FA exposure (Figure 6A); however, the difference was not statistically significant. On the last day of exposure, the AgNP produced 5.2- and 3.9-fold increases in 8-OHdG formation relative to the baseline $8-\mathrm{OHdG}$ levels in the allergic and control mice, respectively $(P<0.01)$. Furthermore, the 8-OHdG levels induced by AgNP inhalation were significantly higher than the levels induced by FA exposure in the allergic and control mice $(P<0.05)$.

\section{Excretion and tissue distribution of AgNP}

AgNP concentrations in the urine and feces were determined over the 7 consecutive days of exposure in the allergic and control mice. The brain, lungs, heart, liver, spleen, and kidney were collected from the allergic and control mice and were analyzed for Ag levels. Significant differences were not observed between the allergic and control mice in the $\mathrm{Ag}$ concentrations in the urine, feces, or tissues (Figure 6B and $\mathrm{C}$ ). The level of $\mathrm{Ag}$ excretion in the urine was insignificant over the 7 day period (17-80 $\mu \mathrm{g} / \mathrm{g}$; Figure 6B), whereas the Ag concentration in the feces decreased significantly 1 day after exposure and was maintained at a stable level after 3 days of exposure (2-21 $\mathrm{g} / \mathrm{g}$; Figure 6B). After 7 days of exposure, the AgNP had accumulated predominantly in the lungs $(17.6 \mu \mathrm{g} / \mathrm{g})$, followed in decreasing order by the heart $(3.6 \mu \mathrm{g} / \mathrm{g})$, spleen $(1.9 \mu \mathrm{g} / \mathrm{g})$, liver $(0.6 \mu \mathrm{g} / \mathrm{g})$, kidneys $(0.3 \mu \mathrm{g} / \mathrm{g})$, and brain $(0.3 \mu \mathrm{g} / \mathrm{g}$; Figure $6 \mathrm{C})$. There was no difference between the AgNP and FA groups regarding the Ag levels in the spleen, liver, kidneys, and brain.

A

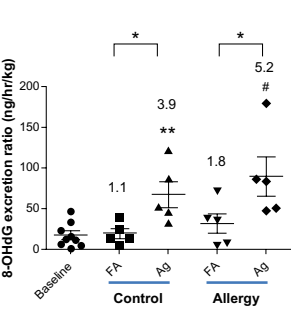

B

C
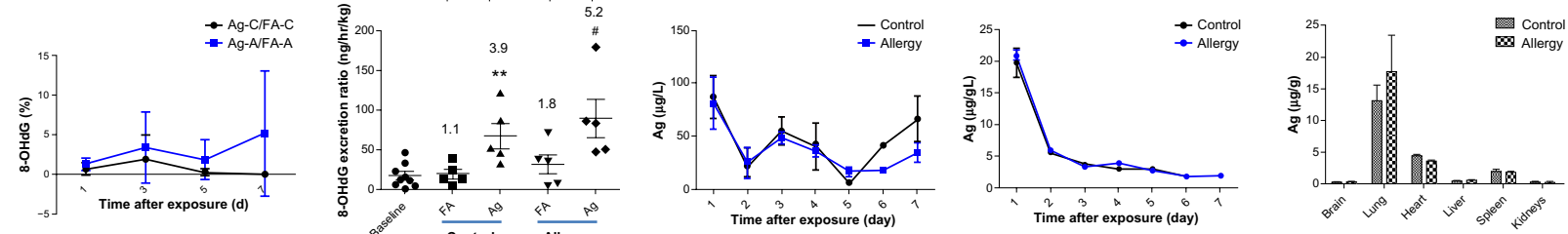

D
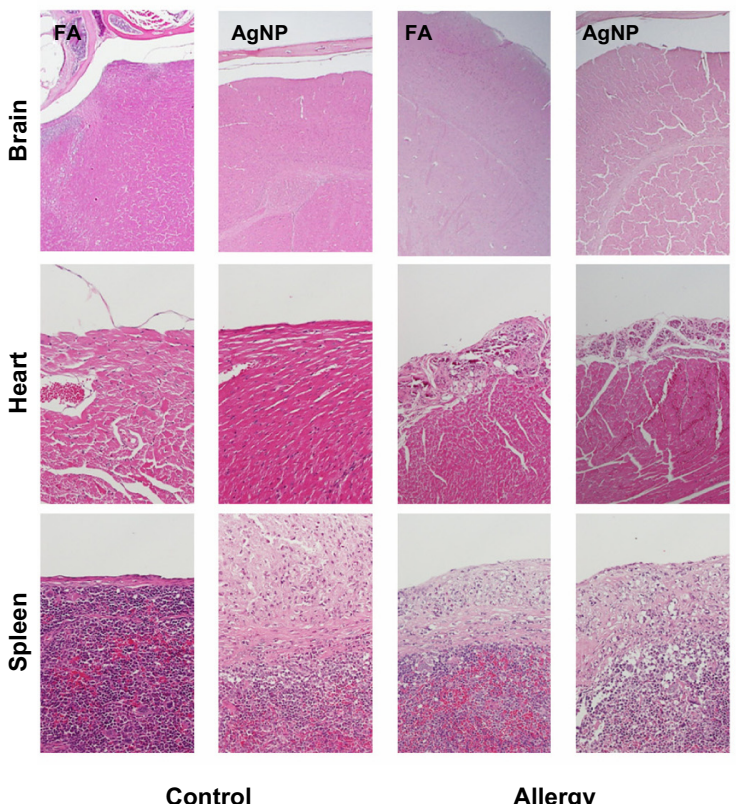

Allergy

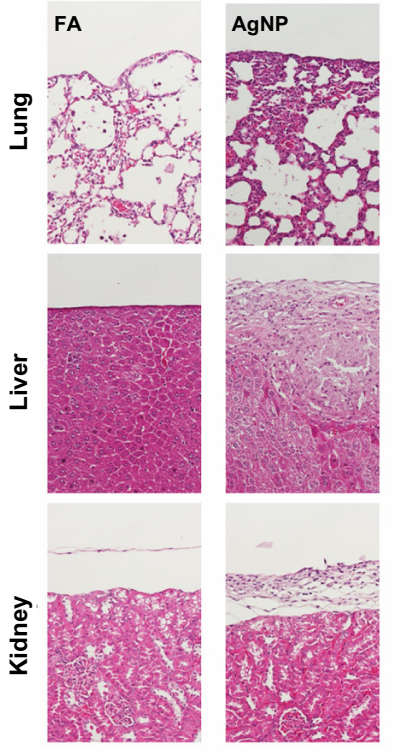

Control

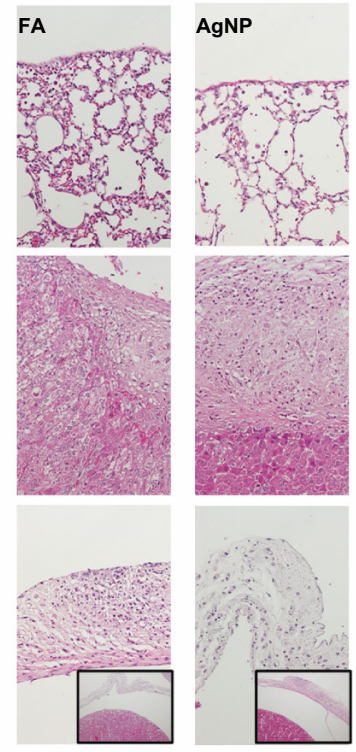

Allergy

Figure 6 (A) Effect of AgNP on the 8-OHdG percentage (ratios of the AgNP exposure group to the FA exposure group; Ag/FA) in the control and allergic mice determined over 7 successive exposure days; comparisons between AgNP/FA in the control and allergic mice with respect to the 8-OHdG excretion ratio (ng/hour/kg) determined on the last day of exposure. (B) AgNP excretion in the urine and feces over the 7 successive days of exposure in the control and allergic mice. (C) Ag distribution in the brain, lungs, heart, liver, spleen, and kidneys after AgNP exposure. (D) Photomicrographs (200x) of hematoxylin and eosin staining of the brain, lungs, heart, liver, spleen, and kidneys from the control and allergic mice after AgNP/FA exposure and OVA challenge. Significant histological changes were not observed in the peri-brain or perilung. Focal dystrophic calcification was observed in the pericardium in the allergic mice exposed to AgNP/FA. Fibrosis with inflammation and dystrophic calcification were observed in the capsule of the liver and spleen of the control mice exposed to AgNP and the allergic mice exposed to AgNP/FA. These mice also demonstrated focal and mild inflammation in the perirenal soft tissue (bottom-right, $20 \times$ for allergic mice).

Notes: Values are expressed as the mean \pm standard deviation; $n=5-6 ; * P<0.001 ; * * P<0.01 ; * P<0.05$

Abbreviations: 8-OHdG, urinary 8-hydroxy-2'-deoxyguanosine; A, antigen; Ag, silver; AgNP, silver nanoparticles; C, control; FA, filtered air; OVA, ovalbumin. 


\section{Histopathology}

Changes in the histology of the brain, lungs, heart, liver, spleen, and kidneys were evaluated after AgNP inhalation and OVA challenge. Importantly, consistent histopathological changes were observed in the peri-tissues. Focal dystrophic calcification was observed in the pericardium of the allergic mice exposed to the AgNP or FA (Figure 6D). Fibrosis accompanied by inflammation and dystrophic calcification was observed in the capsule of the liver and spleen in the control mice exposed to the AgNP and in the allergic mice exposed to the AgNP or FA (Figure 6D). In addition, these mice exhibited focal and mild inflammation in the perirenal soft tissue.

\section{Discussion and conclusions}

To investigate the effects of AgNP in healthy and allergic individuals, the clinical markers of asthma, ie, IgE and IL-13 together with other markers (ie, $\mathrm{LTE}_{4}$ ) suggested by Szefler et al, ${ }^{30}$ were used to determine the allergenicity and toxicity of AgNP generated using the evaporation-condensation method in allergen provocation mouse models. Our results demonstrated that the successive inhalation of AgNP produced a positive allergic response that regulated asthmatic responses, inflammatory responses, and oxidative stress in healthy mice. The asthmatic exacerbation responses partially occurred in the AgNP-exposed mice. In addition, we examined the excretion and accumulation of AgNP in the major tissues and examined the histopathology of these tissues after AgNP inhalation. Our results demonstrated that after inhalation for 7 days, the majority of the AgNP had accumulated in the lungs, with bronchiolocentric interstitial inflammation and inflammatory responses in the pericardium, capsules of the liver and spleen, and perirenal soft tissue, indicating that for nanomedical applications, AgNP inhalation should be used with caution.

Inhalation is a major route for occupational and environmental exposure and is the most relevant route with respect to safety assessment studies. Landsiedel et al suggested the urgency of performing toxicokinetic and inhalation studies to investigate the hazards associated with NP exposure. ${ }^{31}$ The evaporation-condensation method was used to produce AgNP for the investigation of allergic responses after successive inhalation of AgNP in mice, and the principle of the method has been described by Singh et al..$^{20}$ The advantage of this system is that AgNP can be generated continuously and stably for long-term inhalation within a range of levels, reflecting the natural exposure route for respirable NPs rather than exposure via intratracheal instillation or pulmonary aspiration. Moreover, the SEM-EDX showed that the spherical AgNPs generated by this system were uncontaminated, suggesting that the allergenicity and toxicity observed in this study were attributable to the AgNP.

Allergic asthma is a chronic disease broadly defined by inflammation of the airways associated with Penh and mucus hypersecretion..$^{32}$ Penh was determined based on the airway response to methacholine inhalation. Examination of the airway responses to methacholine before and after AgNP inhalation and OVA challenge demonstrated that OVA sensitization alone induced an insignificant alteration in Penh (relative to the responses in the control mice) and significantly increased OVA-specific IgE production in the allergic mice compared to the levels observed before AgNP exposure and OVA challenge. AgNP exposure and OVA challenge induced the highest increase in Penh to methacholine and OVA-specific IgE in the allergic mice compared to the control mice. Therefore, this allergen provocation model is suitable for the evaluation of airway responses after AgNP exposure. Importantly, the Penh was changed moderately by AgNP alone in the control mice or by AgNP/FA in the OVA-sensitized mice. The effect was not observed in the AgNP-treated and OVA-sensitized mice. Previous studies have established that AgNP are causative agents of asthma development, ${ }^{33,34}$ and the disease could be attributed to inflammatory responses. Indeed, our findings also suggested that the exposure of healthy mice to AgNP resulted in an increase in inflammatory reactions (ie, interstitial lymphocytic and neutrophilic inflammatory infiltration in the lungs). However, a previous study showed that AgNP inhalation attenuated airway inflammation and hyperresponsiveness; the contrasting findings could have resulted from the exposure concentration. ${ }^{16}$ Importantly, we observed a mild increase in Penh after consecutive AgNP and OVA challenge in the allergic mice. Moreover, a significant difference was not observed in the lymphocytic and neutrophilic inflammatory infiltration in the lungs of the allergic mice after AgNP or FA inhalation. Additionally, the BALF macrophages decreased after AgNP (allergic and control) and FA exposure (allergic), which was consistent with a previous study. ${ }^{25}$ This decrease may have occurred because the alveolar macrophages became a part of the specific immune response and ingested the AgNP for presentation to lymphocytes. The other important histological finding was the bronchiolocentric interstitial inflammatory responses in the allergic and control mice exposed to the AgNP, which indicates the progression of bronchiolocentric fibrosis. Bronchiolocentric fibrosis is characterized by the pathological pattern of constrictive fibrotic bronchiolitis obliterans, ${ }^{35}$ which is related 
to airflow obstruction. Therefore, we suggest that the AgNP induced the increased airway response and bronchiolocentric inflammation.

The clinical quantification of inflammatory cells in the airway demonstrated that the predominant inflammatory cells responsible for the asthmatic inflammation were eosinophils, T helper (Th) lymphocytes, mast cells, and basophils. ${ }^{3}$ Eosinophilic inflammation of the airways accompanied by an increase in activated and degranulated eosinophils is the phenotype of allergic and nonallergic asthma. ${ }^{36}$ This study demonstrated a significant increase in the recruitment of eosinophils in the lungs of the allergic mice compared to the healthy controls under the same exposure conditions (FA/ AgNP). A previous study investigated the toxicity of metal nanoparticles ${ }^{37}$ and suggested that the allergenicity of nanoparticles may depend on its physicochemical characteristics. Our observation was consistent with the observed levels of OVA-specific IgE, indicating that specific IgE binds to mast cell receptors and initiates a series of membrane events that increase the levels of mediators (eg, histamine). The sequence of cellular events may exacerbate asthma symptoms, as reflected in the allergic reactions. Furthermore, the recruitment of eosinophils in the lungs is primarily controlled by Th lymphocytes and is enhanced by cytokines (eg, IL-13); ${ }^{38}$ infiltration is characteristic of airway inflammation during bronchial asthma. Lymphocytes, including $\mathrm{T}$ and B cells, are considered cells of the adaptive immune system. In allergic reactions (ie, asthma), adaptive immunity requires antigenpresenting cells to stimulate Th2 cell responses. ${ }^{39} \mathrm{Th} 2$ cells produce several cytokines, such as IL-5, which regulates allergen-specific IgE and IL-13, the latter of which is associated with airway responses. ${ }^{39,40}$ Additionally, IL-13 exerts important effects on both structural and inflammatory cells within the airway and has the capacity to initiate the clinical features of airway diseases. ${ }^{41}$ In this study, significantly increased levels of IL-13 were observed in the BALF of the mice exposed to AgNP. Our results indicated that the successive inhalation of AgNP plus OVA challenge resulted in eosinophilic recruitment in the lungs of the healthy control mice, suggesting that AgNP inhalation induces allergic reactions in healthy and allergic mice.

Urinary LTE $_{4}$ is a known marker of cysteinyl leukotriene activity and is associated with allergic inflammation. ${ }^{30}$ In this study, LTE $_{4}$ was used as a supplementary marker for asthmatic reactions, as suggested by Szefler et al. ${ }^{30} \mathrm{LTE}_{4}$ is associated with allergic inflammation, ${ }^{42}$ and this inflammation may be attributed to oxidative stress imbalance. An increasing number of studies have demonstrated the potency of the oxidative stress induced by NP exposure. ${ }^{16,43}$ Continuous AgNP inhalation induced significant $\mathrm{LTE}_{4}$ production in the allergic and control mice. We determined that the AgNP significantly induced the production of systemic oxidative stress in the allergic mice. AgNP inhalation by allergic/healthy mice may increase oxidative stress, which could associate with the increased LTE $_{4}$ and IL-13 levels.

Before AgNP can be applied extensively in nanomedicine, it is important to evaluate their excretion, distribution, and toxicity. This study showed that after sustained exposure for 7 days, few AgNP were excreted via the urinary and digestive systems, and the inhaled AgNP accumulated predominantly in the lungs. Our results suggest that after inhalation, few AgNP are distributed in secondary tissues. Strikingly, the histopathological observations in this study demonstrated that fibrosis and dystrophic calcification were observed primarily in the pericardium, capsules of the liver and spleen, and perirenal soft tissues in the OVA-sensitized mice. These pathological changes occurred in the peripheral regions of the tissues, suggesting that peritoneal inflammation occurred in these mice. The development of fibrosis and dystrophic calcification in the peri-tissues indicates that the intraperitoneal injection of OVA may have caused the inflammatory response in the peritoneum, which led to inflammatory reactions on the surface of these tissues. Notably, the inflammatory responses in the peritoneum were also observed in the control mice after AgNP inhalation; we suspect that this inflammation was caused by the inhalation of AgNP. A previous study demonstrated that $30 \mathrm{mg} / \mathrm{m}^{3}$ of carbon nanotubes reached the subpleura of mice after 6 hours of inhalation and resulted in focal inflammatory cell infiltration. ${ }^{44} \mathrm{~A}$ similar finding was observed in office workers; after inhalation into the lungs, carbon NPs could be transported via the lymphatic and blood vessels, after which they accumulated in the peritoneum. ${ }^{45}$ Our data on AgNP excretion and distribution and the histopathological examination indicated that the exposure of the mice to AgNP allowed focal accumulation of these NPs in the respiratory system, and no significant difference in $\mathrm{Ag}$ levels between the AgNP and FA control mice was detected in the spleen, liver, or kidneys. Therefore, we suggest that the AgNP were transported via the lymph nodes from the lungs to the abdominal cavity and caused peritoneal inflammation. The safety of AgNP in healthy and allergic subjects requires further investigation.

In summary, our study provides a better understanding of the allergenicity and toxicity of AgNP inhalation in asthma. This study suggests that AgNP inhalation has allergic and inflammatory effects in both healthy and allergic mice, 
which exhibited bronchiolocentric inflammatory infiltration. Furthermore, the inhaled AgNP accumulated predominantly in the lungs after 7 days of exposure and induced inflammatory responses in the peritoneum. However, further study regarding the use of house dust mites for the sensitization of mice is required. The allergic responses caused by AgNP synthesized using wet chemical methods should be investigated. Given this uncertainty, precautions that minimize inhalation during AgNP administration would be prudent until further studies can assess the long-term pulmonary and peripheral cytological responses to repeated AgNP exposure. The safety of AgNP in airway therapeutic strategies should be examined carefully before commercial application.

\section{Acknowledgments}

The authors wish to thank DrYuan-Horng Yan, Dr Kuo-Liang Huang, Miss Yin-Jyun Lin, and Miss Karen Cheng for their technical assistance for this research. This study was founded by the National Science Council of Taiwan (grant number 100-2621-M-002-005 and 101-2621-M-002-004) and Taipei Medical University (TMU101-AE1-B53).

\section{Disclosure}

The authors report no conflicts of interest in this work.

\section{References}

1. Global Initiative for Asthma [homepage on the Internet]. Global Strategy for Asthma Management and Prevention 2011. Publisher; 2011. Available from: http://www.ginasthma.org/local/uploads/files/ GINA_Report_March13.pdf. Accessed September 18, 2013.

2. To T, Stanojevic S, Moores G, et al. Global asthma prevalence in adults: findings from the cross-sectional world health survey. BMC Public Health. 2012;12:204.

3. Kazani S, Israel E. Update in asthma 2011. Am J Respir Crit Care Med. 2012;186(1):35-40.

4. Laumbach RJ, Kipen HM. Respiratory health effects of air pollution: update on biomass smoke and traffic pollution. JAllergy Clin Immunol. 2012;129(1):3-11; quiz 12.

5. Brunekreef B, Forsberg B. Epidemiological evidence of effects of coarse airborne particles on health. Eur Respir J. 2005;26(2):309-318.

6. Li N, Wang M, Bramble LA, et al. The adjuvant effect of ambient particulate matter is closely reflected by the particulate oxidant potential. Environ Health Perspect. 2009;117(7):1116-1123.

7. Alberg T, Cassee FR, Groeng EC, Dybing E, Løvik M. Fine ambient particles from various sites in europe exerted a greater IgE adjuvant effect than coarse ambient particles in a mouse model. J Toxicol Environ Health A. 2009;72(1):1-13.

8. Liu H, Yang D, Yang H, et al. Comparative study of respiratory tract immune toxicity induced by three sterilisation nanoparticles: silver, zinc oxide and titanium dioxide. J Hazard Mater. 2013;248-249: 478-486.

9. Lee S, Kim MS, Lee D, et al. The comparative immunotoxicity of mesoporous silica nanoparticles and colloidal silica nanoparticles in mice. Int J Nanomedicine. 2013;8:147-158.

10. Roberts MS, Roberts MJ, Robertson TA, et al. In vitro and in vivo imaging of xenobiotic transport in human skin and in the rat liver. J Biophotonics. 2008;1(6):478-493.
11. Lee CN, Wang YM, Lai WF, et al. Super-paramagnetic iron oxide nanoparticles for use in extrapulmonary tuberculosis diagnosis. Clin Microbiol Infect. 2012;18(6):E149-E157.

12. Szelenyi I. Nanomedicine: evolutionary and revolutionary developments in the treatment of certain inflammatory diseases. Inflamm Res. 2012;61(1):1-9.

13. Cho WS, Duffin R, Howie SE, et al. Progressive severe lung injury by zinc oxide nanoparticles; the role of $\mathrm{Zn} 2+$ dissolution inside lysosomes. Part Fibre Toxicol. 2011;8:27.

14. Beckett WS, Chalupa DF, Pauly-Brown A, et al. Comparing inhaled ultrafine versus fine zinc oxide particles in healthy adults: a human inhalation study. Am J Respir Crit Care Med. 2005;171(10):1129-1135.

15. Chaloupka K, Malam Y, Seifalian AM. Nanosilver as a new generation of nanoproduct in biomedical applications. Trends Biotechnol. 2010;28(11):580-588.

16. Park HS, Kim KH, Jang S, et al. Attenuation of allergic airway inflammation and hyperresponsiveness in a murine model of asthma by silver nanoparticles. Int J Nanomedicine. 2010;5:505-515.

17. Jang S, Park JW, Cha HR, et al. Silver nanoparticles modify VEGF signaling pathway and mucus hypersecretion in allergic airway inflammation. Int J Nanomedicine. 2012;7:1329-1343.

18. Hao M, Comier S, Wang M, Lee JJ, Nel A. Diesel exhaust particles exert acute effects on airway inflammation and function in murine allergen provocation models. J Allergy Clin Immunol. 2003;112(5):905-914.

19. Ho M, Wu KY, Chein HM, Chen LC, Cheng TJ. Pulmonary toxicity of inhaled nanoscale and fine zinc oxide particles: mass and surface area as an exposure metric. Inhal Toxicol. 2011;23(14):947-956.

20. Singh Y, Javier JRN, Ehrman SH, Magnusson MH, Deppert K. Approaches to increasing yield in evaporation/condensation nanoparticle generation. J Aerosol Sci. 2002;33(9):1309-1325.

21. Maciejczyk P, Chen LC. Effects of subchronic exposures to concentrated ambient particles (CAPs) in mice. VIII. Source-related daily variations in in vitro responses to CAPs. Inhal Toxicol. 2005;17(4-5): 243-253.

22. Moreno T, Gibbons W, Jones T, Richards R. The geology of ambient aerosols: characterising urban and rural/coastal silicate PM10-12.5 and PM2.5 using high-volume cascade collection and scanning electron microscopy. Atmos Environ. 2003;37(30):4265-4276.

23. Pichavant M, Goya S, Hamelmann E, Gelfand EW, Umetsu DT. Animal models of airway sensitization. Curr Protoc Immunol. 2007; Chapter 15:Unit 15.18.

24. Jang AS, Choi IS, Lee JH, Park CS, Park CS. Prolonged ozone exposure in an allergic airway disease model: adaptation of airway responsiveness and airway remodeling. Respir Res. 2006;7:24.

25. Gavett SH, Haykal-Coates N, Copeland LB, Heinrich J, Gilmour MI. Metal composition of ambient PM2.5 influences severity of allergic airways disease in mice. Environ Health Perspect. 2003;111(12): 1471-1477.

26. Wan GH, Li CS, Lin RH. Airborne endotoxin exposure and the development of airway antigen-specific allergic responses. Clin Exp Allergy. 2000;30(3):426-432.

27. Micheletto C, Tognella S, Visconti M, Trevisan F, Dal Negro RW. Changes in urinary LTE4 and nasal functions following nasal provocation test with ASA in ASA-tolerant and -intolerant asthmatics. Respir Med. 2006;100(12):2144-2150.

28. Jones T, Moreno T, BéruBé K, Richards R. The physicochemical characterisation of microscopic airborne particles in south Wales: a review of the locations and methodologies. Sci Total Environ. 2006;360(1-3): 43-59.

29. Sitkauskiene B, Rådinger M, Bossios A, Johansson AK, Sakalauskas R, Lötvall J. Airway allergen exposure stimulates bone marrow eosinophilia partly via IL-9. Respir Res. 2005;6:33.

30. Szefler SJ, Wenzel S, Brown R, et al. Asthma outcomes: biomarkers. J Allergy Clin Immunol. 2012;129(Suppl 3):S9-S23.

31. Landsiedel R, Ma-Hock L, Haussmann HJ, van Ravenzwaay B, Kayser M, Wiench K. Inhalation studies for the safety assessment of nanomaterials: status quo and the way forward. Wiley Interdiscip Rev Nanomed Nanobiotechnol. 2012;4(4):399-413. 
32. Kim SH, Hong JH, Lee YC. Ursolic acid, a potential PPAR $\gamma$ agonist, suppresses ovalbumin-induced airway inflammation and Penh by downregulating IL-5, IL-13, and IL-17 in a mouse model of allergic asthma. Eur J Pharmacol. 2013;701(1-3):131-143.

33. Xu Y, Tang H, Liu JH, Wang H, Liu Y. Evaluation of the adjuvant effect of silver nanoparticles both in vitro and in vivo. Toxicol Lett. 2013;219(1):42-48.

34. Bezemer GF, Bauer SM, Oberdörster G, et al. Activation of pulmonary dendritic cells and Th2-type inflammatory responses on instillation of engineered, environmental diesel emission source or ambient air pollutant particles in vivo. J Innate Immun. 2011;3(2):150-166.

35. Cordier JF. Challenges in pulmonary fibrosis. 2: Bronchiolocentric fibrosis. Thorax. 2007;62(7):638-649.

36. Chung KF. Inflammatory biomarkers in severe asthma. Curr Opin Pulm Med. 2012;18(1):35-41.

37. Cho WS, Duffin R, Poland CA, et al. Differential pro-inflammatory effects of metal oxide nanoparticles and their soluble ions in vitro and in vivo; zinc and copper nanoparticles, but not their ions, recruit eosinophils to the lungs. Nanotoxicology. 2012;6(1):22-35.

38. Maes T, Provoost S, Lanckacker EA, et al. Mouse models to unravel the role of inhaled pollutants on allergic sensitization and airway inflammation. Respir Res. 2010;11:7.
39. Lin KW, Li J, Finn PW. Emerging pathways in asthma: innate and adaptive interactions. Biochim Biophys Acta. 2011;1810(11): $1052-1058$

40. Wills-Karp M. The gene encoding interleukin-13: a susceptibility locus for asthma and related traits. Respir Res. 2000;1(1):19-23.

41. Brightling CE, Saha S, Hollins F. Interleukin-13: prospects for new treatments. Clin Exp Allergy. 2010;40(1):42-49.

42. Paruchuri S, Tashimo H, Feng C, et al. Leukotriene E4-induced pulmonary inflammation is mediated by the P2Y12 receptor. J Exp Med. 2009;206(11):2543-2555.

43. Mei N, Zhang Y, Chen Y, et al. Silver nanoparticle-induced mutations and oxidative stress in mouse lymphoma cells. Environ Mol Mutagen. 2012;53(6):409-419.

44. Ryman-Rasmussen JP, Cesta MF, Brody AR, et al. Inhaled carbon nanotubes reach the subpleural tissue in mice. Nat Nanotechnol. 2009;4(11):747-751.

45. Theegarten D, Boukercha S, Philippou S, Anhenn O. Submesothelial deposition of carbon nanoparticles after toner exposition: case report. Diagn Pathol. 2010;5:77.
International Journal of Nanomedicine

\section{Publish your work in this journal}

The International Journal of Nanomedicine is an international, peerreviewed journal focusing on the application of nanotechnology in diagnostics, therapeutics, and drug delivery systems throughout the biomedical field. This journal is indexed on PubMed Central, MedLine, CAS, SciSearch $\AA$, Current Contents ${ }^{\circledR} /$ Clinical Medicine,

\section{Dovepress}

Journal Citation Reports/Science Edition, EMBase, Scopus and the Elsevier Bibliographic databases. The manuscript management system is completely online and includes a very quick and fair peer-review system, which is all easy to use. Visit http://www.dovepress.com/ testimonials.php to read real quotes from published authors. 